\title{
Variations in the Glycemic Response to Carbohydrates: Do High Responders Have a Special Benefit of Using Low Glycemic Foods?
}

\begin{abstract}
Arne T. Høstmark*
University of Oslo, Norway, Section of Preventive Medicine and Epidemiology, Box 1130 Blindern, 0318 Oslo, Norway

Abstract: The postprandial blood glucose elevation (PPG) seems to be an important risk factor for diabetes Type 2 and coronary heart disease. We have tested whether high responders have a greater PPG reduction than low responders when changing from high to low glycemic foods, as might be anticipated. In 25 healthy, young men the glycemic effects of six different, low glycemic foods (each ingested in portions providing $50 \mathrm{~g}$ carbohydrates) were determined and compared with the glycemic response after intake of $50 \mathrm{~g}$ glucose. The reduction in glycemia (delta incremental area under the curve) was proportional to the glycemic response after ingesting glucose $(r>0.8 ; p \leq 0,001)$. Thus, high responders to ingested glucose might have a special benefit of using low glycemic foods.
\end{abstract}

Keywords: Blood glucose, carbohydrate meal, high responders, men.

\section{INTRODUCTION}

The magnitude and duration of postprandial blood glucose elevations seem to be important risk factors for obesity, diabetes Type 2 and coronary heart diseases [1,2]. For preventive medicine it would seem desirable to avoid large postprandial elevations of the blood glucose concentration.

Traditionally, carbohydrates are classified into simple and complex ones, but this classification does not give information about their glycemic effects [3]. The glycemic food effect can be estimated by the glycemic index (GI), which is defined as the Incremental Area Under the blood glucose Curve (IAUC) after intake of 50g carbohydrate in a test food as per cent of the IAUC after ingesting $50 \mathrm{~g}$ glucose $[4,5]$. The following cut-off limits between low, middle and high GI have been proposed: $<55 \%$ (low), 55-70 \% (medium), > $70 \%$ (high) [6]. Conceivably, also the amount of carbohydrate ingested influences the glycemic effect. Suggested cut-off limits [7] for the glycemic load (GL) of a portion, i.e. the product of GI and amount carbohydrate in the portion: $\leq 10 \mathrm{~g}$ (low), 11-19 $\mathrm{g}$ (medium), and > $19 \mathrm{~g}$ (high).

The standard deviations of the GI and GL are high, presumably due to great inter- individual variations. It might be suggested that the reduction in the glycemic effect after ingesting a low glycemic food might be related to the subject's maximal glycemic response, as expressed either in absolute or relative terms. Accordingly, in the present work we ask the question: Is there a relationship between the IAUC values after ingesting pure glucose and the reductions in IAUC (delta IAUC) observed after ingesting different low glycemic foods containing the same amount of carbohydrate? The reductions in glycemic effect were expressed in relative and absolute terms.

\section{MATERIALS AND METHODOLOGY}

After approval (S-02013) by the Regional Ethics Committee of Norway-South, on separate days the postprandial

*Address correspondence to this author at the University of Oslo, Norway, Section of Preventive Medicine and Epidemiology, Box 1130 Blindern, 0318 Oslo, Norway; Tel: +47 228582 84; Fax: +47 228582 80; E-mail: a.t.hostmark@medisin.uio.no blood glucose response to ingesting $50 \mathrm{~g}$ glucose was studied, with 3 repeats, as well as 6 food trials in altogether 25 young (18-40 years) healthy men, non-smokers, in the post absorptive state. For each food 10 men participated, but some of the men participated in more than one food trial; two of the men participated in 3 of the 6 trials. Having fasted for 10 hours the subjects ingested either $50 \mathrm{~g}$ glucose dissolved in $200 \mathrm{ml}$ water, or an amount of different foods providing $50 \mathrm{~g}$ carbohydrates; $200 \mathrm{ml}$ water was ingested as well. The foods were: \#1 fettuccine, \#2 coarse rye bread, \#3 bread of whole grain, \#4 pizza, and \#5 and \#6 were two types of baguette. Food amounts providing $50 \mathrm{~g}$ carbohydrates in diets \#1-6 were: 116, 246, 275, 224, 376 and $369 \mathrm{~g}$.

Capillary blood glucose was determined on a HemoCue Glucose 201+ instrument). The Incremental Area Under the Curve (IAUC) was calculated, using the trapezoidal rule (5). Significance of differences between IAUC values after the foods and glucose intake was assessed by paired t-test, and repeated measure ANOVA, using SPSS. Pearson's correlation coefficients were used to test the relationship between IAUC for $50 \mathrm{~g}$ glucose and the reduction in IAUC when ingesting the various foods. Mean values \pm SEM are shown on the blood glucose curves. For GI-values, mean values with $95 \%$ confidence intervals are presented. $\mathrm{P}<0.05$ was considered statistically significant. The figures were produced by SigmaPlot.

\section{RESULTS}

The blood glucose curves after intake of $50 \mathrm{~g}$ glucose increased in the time period 0 to $45 \mathrm{~min}$, reached a peak value after $45 \mathrm{~min}$ and then decreased progressively to values slightly below the fasting value by the end of the observation period of $2 \mathrm{~h}$ (Fig. 1). The blood glucose curve after ingesting the various foods started at a similar level as after ingesting glucose, increased until about $45 \mathrm{~min}$, but the curve was less steep than after glucose intake, implying that the blood glucose excursions were attenuated. The blood glucose responses to intake of $50 \mathrm{~g}$ pure glucose, and $50 \mathrm{~g}$ carbohydrate in the various foods were significantly different $(\mathrm{P}<0.01)$ for all of the foods. GI for the different foods, i.e. (IAUC-food/IAUC-glucose)*100 were $38 \pm 3(32-44) \%$ 

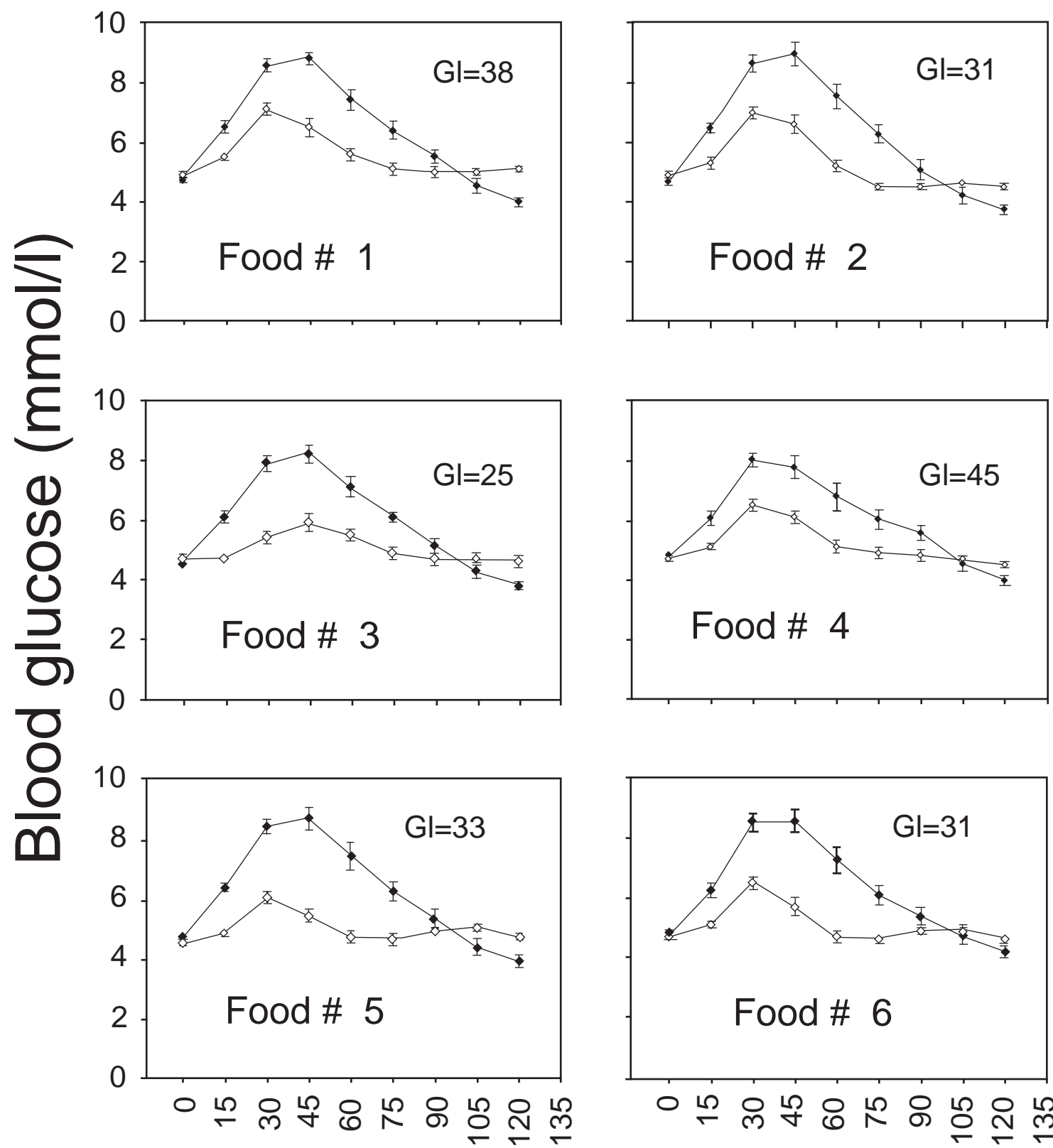

\section{Time (min)}

Fig. (1). Blood glucose values in healthy, young men after intake of 6 different foods, with an amount providing $50 \mathrm{~g}$ carbohydrates, and ingested in the time period 0-15 min (open circles), compared with the blood glucose response after ingesting $50 \mathrm{~g}$ pure glucose, in the same subjects (filled circles). Food \#1 to 6 was: fettuccine, coarse rye bread, whole grain bread, pizza, and two types of baguette (food \#5 and 6 ). Food amounts providing $50 \mathrm{~g}$ carbohydrates in diets \#1-6 were: 116, 246, 275, 224, 376 and $369 \mathrm{~g}$. Mean values $\pm \mathrm{SEM}$ ( $\mathrm{n}=10$ in all groups, with a total $\mathrm{N}=25$, since some of the test subjects participated in more than one trial). In all panels there was a significant ( $\mathrm{p}<0.01)$ main effect of time, and interaction between time and food type (repeated measure ANOVA).

(mean \pm SEM, 95\% confidence interval), $30 \pm 4$ (22-38), 25 \pm

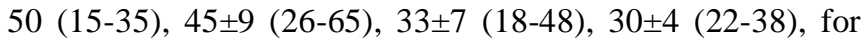
food \#1-6, respectively. Thus, all these foods were low glycemic ones, as judged from the criteria of Monro [5].

There was no consistent relationship between IAUC for glucose and the percentage change from this value for any of the different foods (not shown). In contrast, the IAUC-value after ingesting $50 \mathrm{~g}$ glucose was proportional to the individ- ual absolute IAUC-reduction when ingesting the lower glycemic foods, with correlation coefficients of above 0.8 and p $\leq 0,001$ (Fig. 2).

\section{DISCUSSION}

These results suggest that variations in GI values not only reflect the carbohydrate type and amount ingested, but also to a great extent can be attributed to inter-individual 


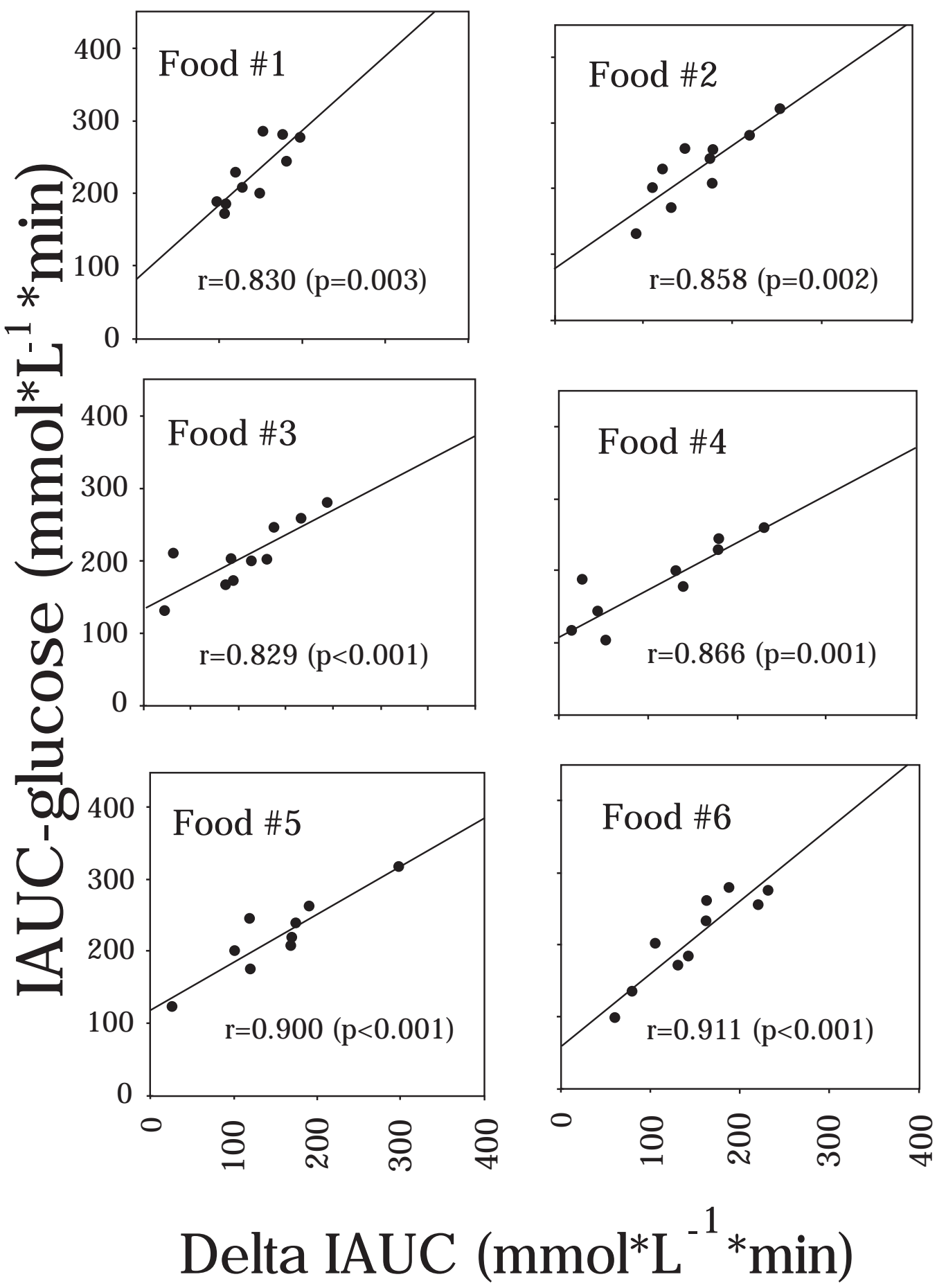

Fig. (2). Scatter plot of the relationship between the individual absolute IAUC-reduction (Delta-IAUC) elicited by intake of $50 \mathrm{~g}$ carbohydrate in 6 low glycemic foods as compared with $50 \mathrm{~g}$ glucose, and the corresponding IAUC after ingestion of $50 \mathrm{~g}$ glucose, in healthy young men. Food \#1 to 6 was: fettuccine, coarse rye bread, whole grain bread, pizza, and two types of baguette (food \#5 and 6). Food amounts providing $50 \mathrm{~g}$ carbohydrates in diets \#1-6 were: 116, 246, 275, 224, 376 and 369g. Pearson's correlation coefficients and p-values are shown in each of the panels. Number of subjects in each trial $=10$; total $\mathrm{N}=25$, since some of the test subjects participated in more than one trial.

variations in the glycemic response. However, the study did not focus upon mechanisms behind the variations, which could be related to variations in the digestion of various carbohydrate containing foods, and the intestinal uptake rate of glucose.
Although the material is small, the results seem to favor the idea that there are low and high responders in the population as regards postprandial blood glucose elevation. Three of the subjects participated in 2 of the 6 food trials, and were for each food in the highest glycemic response tertile. Con- 
versely, 3 other subjects were low responders in 2 food trials, and one subject was low responder for 3 foods. These observations would seem to indicate consistency in a person's glycemic response pattern.

Possibly, the results might have a preventive message: if a subject is a high responder to ingested carbohydrates, i.e. has a high glycemic response after ingesting glucose and other high glycemic foods, then this person would probably also have a great reduction in the postprandial glycemic response if changing to low glycemic foods.

\section{CONCLUSION}

High responders to ingested glucose might have a special benefit of using low glycemic foods.

\section{ACKNOWLEDGEMENT}

The author gratefully acknowledge the technical assistance of Eva Kristensen.

\section{REFERENCES}

[1] Morris KL, Zemel MB. Glycemic index, cardiovascular disease, and obesity. Nutr Rev 1999; 57: 261-73.

[2] Gugliano D, Ceriello A. The Toxic trio: Acute, chronic, and postprandial hyperglycemia. Nutr Metab Cardiovasc Dis 2001; 11: 803 .

[3] Foster-Powell K, Holt SH, Brand-Miller JC. International table of glycemic index and glycemic load values: 2002. Am J Clin Nutr 2002; 76: 5-56

[4] Jenkins DJ, Wolever TM, Taylor RH, et al. Glycemic index of foods: a physiological basis for carbohydrate exchange. Am J Clin Nutr 1981; 34: 362-66.

[5] WHO/FAO: Carbohydrates in Human Nutrition. Report of a joint FAO /WHO expert consultation on carbohydrates in human nutrition. Paper 66, 1997.

[6] Monro JA. Glycaemic glucose equivalent: combining carbohydrate content, quantity and glycaemic index of foods for precision in glycaemia management. Asia Pacific J Clin Nutr 2002; 11: 217-22.

[7] Brand- Miller JC, Holt SHA. Letter to the editor. Am J Clin Nutr 2003; 77: 994-5. 